\title{
Endoscopic repair of CSF rhinorrhea through persistent Sternberg's canal in an elderly patient with ankylosing spondylitis
}

\author{
Deepak Khatri, Suyash Singh, Kuntal Kanti Das, AwadheshKumar Jaiswal* and Raj Kumar \\ Department of Neurosurgery, Sanjay Gandhi Postgraduate Institute of Medical Sciences, Lucknow, India
}

A 69-year-old gentleman with ankylosing spondylitis presented with spontaneous clear watery discharge from his right nostril for 2 months without anosmia. There was no history of preceding trauma or features suggesting benign intracranial hypertension. Neurological examination was essentially normal. Considering a diagnosis of spontaneous CSF rhinorrhea, we ordered plain computed tomography of the head including a CT cisternogram. A bony defect in the lateral wall of the sphenoid sinus was unravelled. Endoscopic endonasal transethmoidal trans-sphenoidal approach was taken striking a balance of optimum surgical position and the fixed neck and trunk deformity

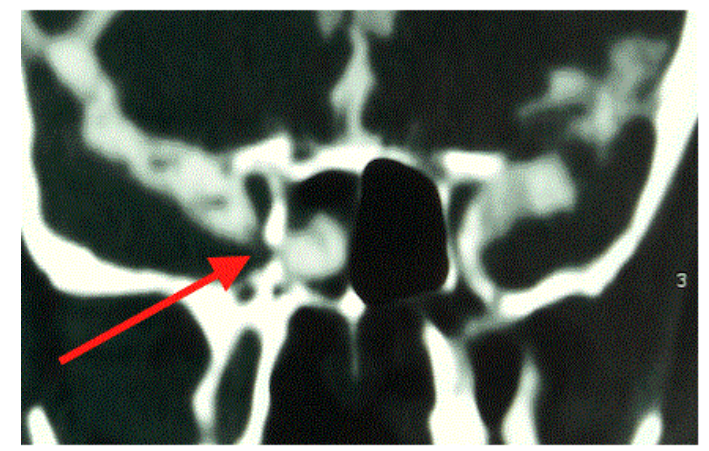

Figure 1. Coronal section of the CT cisternogram reveals a defect in the right lateral wall of the sphenoid sinus through which a CSF pouch (pseudomeningocele) is projecting in to the right half of the sphenoid sinus. The left hemisinus is normally aerated and there is no evidence of enlarged ventricles.

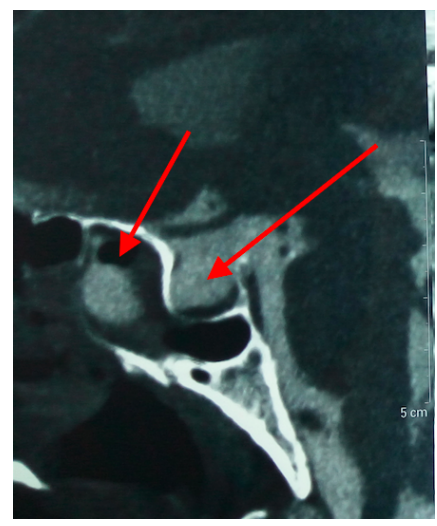

Figure 2. Sagittal section of the CT cisternogram reveals CSF intensity inside the sella suggesting an empty sella while the pseudomeningocele is apparent on the lateral wall of the sphenoid sinus. The sphenoid sinus cavity is partially aerated indicating presence of either mucosal edema or collection. of the patient. The defect was repaired with fat and fascia lata graft harvested from the thigh. CSF leak completely subsided after the repair. The patient recovered uneventfully after surgery and is doing well at follow-up.

Ommaya proposed an etiological classification of CSF leaks through the anterior skull base into Spontaneous, Traumatic and Pathological $[1,2]$. Of all possible sites of spontaneous CSF leaks, lateral wall of the sphenoid sinus (persistent Sternberg's canal) remains infrequently encountered in clinical practice. Embryologically, the Sternberg's canal represents defective fusion between the posterior basisphenoid and lateral part of greater wing leading to abnormal communication of the cavity of the sphenoid sinus with the intracranial space [3]. Clinically, patients with CSF leaks secondary to a sphenoid sinus defect are differentiated from the more common cribriform plate defects by having a predominant post nasal drip than frank rhinorrhea [4].

The evaluation of a patient with CSF rhinorrhea involves different tests for confirming the nature of the nasal discharge and various imaging studies to detect the putative site of CSF leak. The initial management in spontaneous CSF rhinorrhea remains a trial of conservative treatment for 2-4 weeks. If the leak still persists, surgical intervention is warranted. Endoscopic transnasal approach provides a minimally invasive means of sealing the defect and is currently very popular all across the world [4].

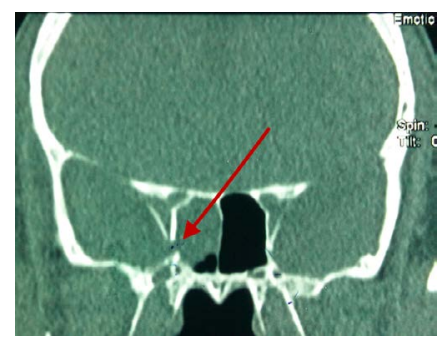

Figure 3. Coronal section of the plain CT head in bone window shows the well-defined bony defect in the right lateral wall of the sphenoid sinus abutting the roots of the pterygoid plates.

The right hemisinus is opacified.

Correspondence to: Awadhesh Kumar Jaiswal, Professor, Department of Neurosurgery, Sanjay Gandhi Postgraduate Institute of Medical Sciences, Lucknow, Uttar Pradesh, India, E-mail: akjaiswal@sgpgi.ac.in

Received: November 10, 2016; Accepted: November 16, 2016; Published: November 18, 2016 


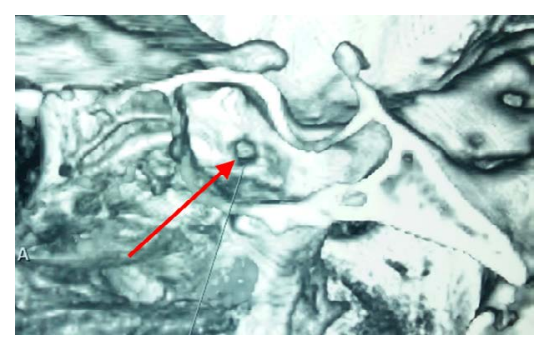

Figure 4. Sagittal reconstructed plain CT shows the small lateral wall defect. The margins are irregular, probably secondary to the persistent CSF pulsations transmitted in to the pseudomeningocele.

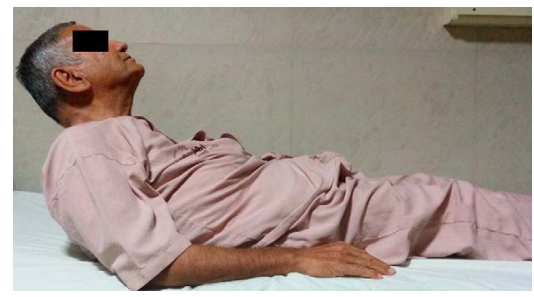

Figure 5. Shows the position of the patient when he tried to lie supine. The pathological fusion of the spine did not allow the patient to assume a supine position leading to a persistent propped up position. This led to our inability to position him for classical transnasal endoscopic surgery. We had to resort to fibreoptic intubation and operate in this position with innovative manipulations of the endoscope.

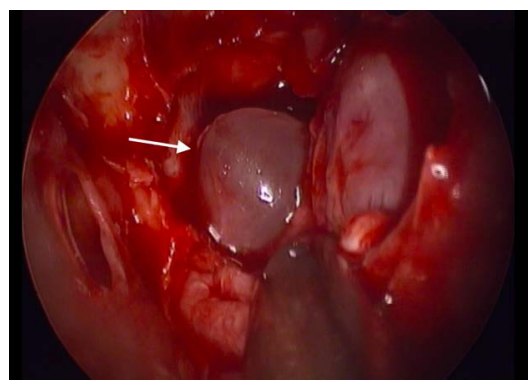

Figure 6. Shows the endoscopic view of the pseudomeningocele protruding into the sphenoid sinus through a lateral wall defect (left hand side of the image). On the right side of the image, there lies the nasal septum while the suction tip represents the inferior aspect towards the nasal floor. Only right half of the sphenoid sinus was opened leaving the left half completely untouched.

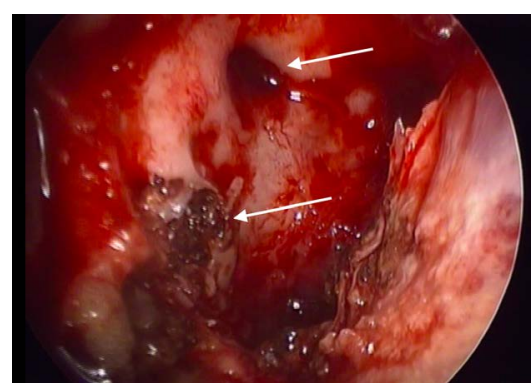

Figure 7. Shows the bony defect seen during surgery (pointed with an arrow). The pseudomeningocele was excised and the bony defect was clearly defined. The bone surrounding the defect was made bare, a key step for successful surgical repair. The depression seen above the bony defect represents the lateral optico-carotid recess, the internal view of the pneumatized optic strut.

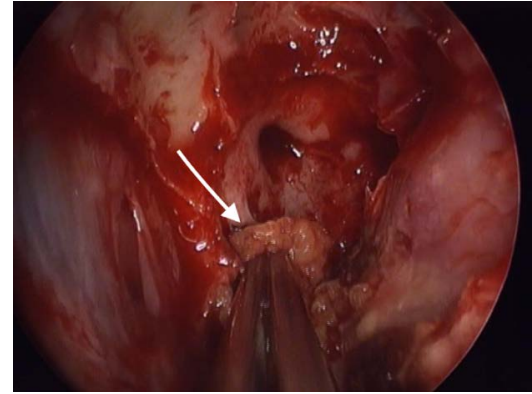

Figure 8. Shows plugging of the bony defect with fat procured from right thigh. The fat was plugged inside the defect so that part of the fat projected on either side of the defect (bath plug technique). This plugging is very important for successful repair.

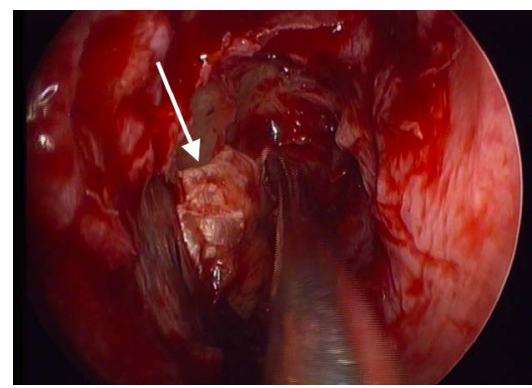

Figure 9. Shows coverage of the fat plugged defect with fascia lata graft. The fascial graft was laid over the defect ensuring coverage of the adjoining bare bone. This initial layering was supplemented by gel foams and surgicel. The ipsilateral nasal cavity was packed (for 48 hours) to provide temporary support to the repair.

\section{References}

1. Ommaya AK, Di Chiro G, Baldwin M, Pennybacker JB (1968) Non-traumatic cerebrospinal fluid rhinorrhoea. J Neurol Neurosurg Psychiatry 31: 214-225.

2. Yadav YR, Parihar V, Janakiram N, Pande S, Bajaj J, et al. (2016) Endoscopic management of cerebrospinal fluid rhinorrhea. Asian J Neurosurg 11: 183-193. [Crossref]

3. Barañano CF, Curé J, Palmer JN, Woodworth BA (2009) Sternberg's canal: fact or fiction? Am J Rhinol Allergy 23: 167-171. [Crossref]

4. Komotar RJ, Starke RM, Raper DM, Anand VK, Schwartz TH (2013) Endoscopic endonasal versus open repair of anterior skull base CSF leak, meningocele, and encephalocele: a systematic review of outcomes. J Neurol Surg A Cent Eur Neurosurg 74: 239-250. [Crossref]

Copyright: (C2016 Khatri D. This is an open-access article distributed under the terms of the Creative Commons Attribution License, which permits unrestricted use, distribution, and reproduction in any medium, provided the original author and source are credited. 